\title{
NOTES
}

BRIEF contributions in any field of instrumentation or technique within the scope of the journal should be submitted for this section. Contributions should in general not exceed 500 words.

\section{Detector system with high time resolution for the continuous measurement of spectra in the vacuum ultraviolet wavelength range}

\author{
W. Biel, G. Bertschinger, and the TEXTOR Team \\ Institut für Plasmaphysik, Forschungszentrum Jülich GmbH, EURATOM Association, Trilateral Euregio \\ Cluster, D-52425 Jülich, Germany
}

(Received 7 February 2003; accepted 3 April 2004; published online 29 June 2004)

\begin{abstract}
A new detector system with high time resolution $(1 \mathrm{~ms})$ has been developed and applied for the continuous measurement of spectra in the vacuum ultraviolet (VUV) and extreme ultraviolet (EUV) wavelength region at the fusion plasma experiment Torus Experiment for Technology-Oriented Research (TEXTOR). The system consists of an open multichannel-plate (MCP) detector with subsequent first generation (Gen I) light amplifier and a camera head which is based on a linear photodiode array with 1024 elements (pixels). The camera head provides the output signals of the individual pixels sequentially as an analog voltage with a full spectra rate of 1000 per second, which are measured using a PC-based data acquisition system. Three vacuum spectrometers operating in the VUV/EUV region $(10-130 \mathrm{~nm})$ have been equipped with the new system and a successful campaign of measurements from about 4000 discharges at TEXTOR has been performed. Spectra are recorded with a usable linear dynamic range of 10 bit and a wavelength resolution corresponding to a width of 3-4 pixels. (C) 2004 American Institute of Physics. [DOI: 10.1063/1.1763261]
\end{abstract}

In magnetically confined fusion plasmas a detailed monitoring of the impurity content is frequently performed using broadband spectrometers in the vacuum ultraviolet (VUV) and extreme ultraviolet wavelength range. ${ }^{1-5}$ Operating these spectrometers at high time resolution, the radial transport properties of plasma impurities can be studied by means of transient impurity injection experiments, where the time evolution of spectral lines from different ionization stages is used to observe the radial propagation of impurity particles from the cold plasma edge towards the hot plasma center. $^{2,6-11}$ In the case of medium-sized fusion experiments like the tokamak TEXTOR, an accurate analysis of such transient experiments requires a time resolution on the order of $1 \mathrm{~ms}$, since the typical radial particle transport times are in the range of only 5-30 ms. ${ }^{11}$ In this Note we present the design of a detector system based on the Hamamatsu C3904-F linear array which allows to continuously record spectra in the VUV/EUV wavelength range at a full spectra rate of 1000 per second.

The schematics of the detector setup is shown in Fig. 1. An open MCP detector with $40 \mathrm{~mm}$ diameter converts the VUV photons to visible light. The single-stage MCP with high quantum efficiency (CsJ coating), high amplification of up to $10^{4}(\mathrm{~L} / \mathrm{D}$ ratio of 60/1) and extended dynamic range allows for linear operation up to maximum output current densities of about $j_{\text {output }}=0.5 \mu \mathrm{A} / \mathrm{cm}^{2}$, while a fast P46 phosphor ensures high time resolution (decay time below $1 \mu \mathrm{s})$. In order to further increase the detector output light intensity above the level defined by the saturation threshold of the MCP, a proximity-focused first generation (Gen I) light amplifier (by Proxitronic) is used, providing an additional amplification factor of 10. Including all amplification and efficiency factors as well as the transmission losses in the detector setup, an individual electron event at the MCP input side leads to a maximum accumulated charge in the C3904-F linear array of about $q \approx 2.6 \times 10^{5} e^{-} \approx 0.04 \mathrm{pC}$, which is $0.16 \%$ of the full capacity of the pixels $(25 \mathrm{pC})$. The usable linear range of the detector is limited by the saturation threshold of the MCP corresponding to a value of $20 \mathrm{pC} / \mathrm{ms}$ per pixel.

The dimensions of the linear photodiode array (Hamamatsu S3904-F) with 1024 pixels of $2.5 \mathrm{~mm}$ height and $25 \mu \mathrm{m}$ width each are similar to those of the widely used Reticon array, ${ }^{3-5,8,12-16}$ which is however no longer

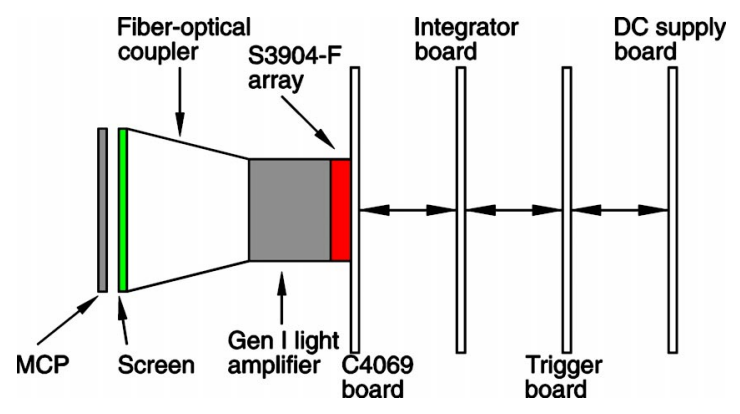

FIG. 1. (Color online) Scheme of the detector setup. 
available. In combination with a C4069 supply board by Hamamatsu, the S3904-F array can be continuously operated at pixel rates of up to $2 \mathrm{MHz}$ and therefore at full spectra rates of up to nearly 2000/s. The C4069 board has recently been replaced by Hamamatsu with a more compact C7883 board, which is functionally equivalent to the former version.

The C4069 board handles the triggering of the linear array and provides the amplified collected charges of the individual pixels. In order to operate the 1024 pixel array with the $\mathrm{C} 4069$ board at a spectra rate of 1000/s, one "master start" input clock signal with frequency $1 \mathrm{kHz}$ and a second synchronous input "master clock" pulse train with frequency $6.55 \mathrm{MHz}$ and with six pulses per pixel are supplied to the C4069 board. On the analog part of the C4069 board a current-to-voltage conversion of the pixel charges is performed, followed by a subsequent analog subtraction of signals from dummy pixels for noise reduction. At the output connector of the C4069 board the analog signals are available in form of sequential short output voltage pulses (FWHM width $100 \mathrm{~ns}$, separation $916 \mathrm{~ns}$ ), where the time integral of the individual pulse is proportional to the collected charge of the pixel. An additional analog switched integrator and amplifier board has been developed and installed in order to convert these short pulses into a smooth analog signal with an amplitude proportional to the time integral of the individual pulse, which is appropriate to be digitized using a standard data acquisition board ("data logger"). The principle of the layout of the integrator board is shown in Fig. 2.

An inverting amplifier based on the OPA 655 operational amplifier OP1 (bandwidth $400 \mathrm{MHz}$ ) serves to adapt the incoming signal level. Resistors R1 (1 k 2 ) and R2 (1-5 k $\Omega$ ) set an amplification factor between 1 and 5 . The subsequent integrator stage utilizes a second OPA 655 operational amplifier OP2 together with capacitor $\mathrm{C} 1(162 \mathrm{pF})$ and resistor R3 $(300 \Omega)$ in order to integrate the incoming signal with a rise time of about $50 \mathrm{~ns}$. Resistor R5 included into the integrator stage prevents the OP2 circuit from ringing. This resistor has no effect on the operation of the integrator since the integrator exit is only connected to the high-impedance input stage of the subsequent EL 2003 driver. The fast analog switch DG 612 (switching time $12 \mathrm{~ns}$ ) is used to discharge the integrator prior to the arrival of the next pixel pulse. A serial resistor R4 (88 $\Omega$ ) limits the discharging current to a value below the maximum specified current for the switch (30 mA). The internal resistance of the DG 612 switch $(18 \Omega)$ together with the external resistor R4 and the integrator capacitor $\mathrm{C} 1$ define a typical 1/e discharging time of about $17 \mathrm{~ns}$, so that a complete discharging $\left(Q_{1} / Q_{0}\right.$ $<10^{-3}$ ) is obtained after about $100 \mathrm{~ns}$. The integrated signal is provided to the output connectors by an EL 2003 driver, which is able to feed the output voltage into a long cable with $50 \Omega$ impedance and $50 \Omega$ termination. Adjusting the offset at OP1 to zero voltage, the use of a separate hold switch in the integrator stage becomes unnecessary and a flat output signal phase with a length of several $100 \mathrm{~ns}$ can be achieved, before the capacitor is being discharged prior to the next input voltage pulse. The remaining offset level mea- sured by the DAQ system can easily be separated from the signals by numerical subtraction. A separate trigger board in the camera head includes a potential free input stage for the incoming "master start" and "master clock" signals via optocouplers and provides these signals to the C4069 board. Furthermore, the trigger board receives the trigger output signal from the C4069 board and provides this signal via adjustable delays to both the DG 612 switch as well as to an EL 2003 driver stage, which feeds the signal to an external $\mathrm{BNC}$ connector for the triggering of the data aquisition system.

For the measurement of the analog camera signals, the T112-4 data acquisition board (by Imtec) with PCI interface is used together with the "INSIGHT" DAQ software by Imtec, operated on a personal computer with a CPU clockrate of $600 \mathrm{MHz}$. This 12 bit DAQ system provides four channels with input voltage ranges selectable up to $10 \mathrm{~V}$ and data rates up to $1.25 \mathrm{MHz}$ each. A common external trigger input allows to operate the system with external triggering synchronous to the camera pixel rate and with parallel measurement of the data from up to four camera heads simultaneously. Using a predefined length of the data sets of 10 megasamples per channel, a continuous readout of measured spectra from four channels is performed over a time of about $10 \mathrm{~s}$, which is well adapted to the duration of the typical plasma discharges at TEXTOR (5-8 s). Since the digital data are transferred directly to the RAM memory of the PC, the possible duration of the measurement is only limited by the size of the PC RAM. A more recent version of the DAQ system based on a faster computer and new driver software can directly shift the data from the DAQ board to the hard disk of the PC and thus allows for long-term measurements (hours) at the full spectra rate of 1000/s. For all measurements presented here, the voltage range of the DAQ system is set to 0 to +5 V. Since the output signals of the OPA 655 operational amplifier saturate at a level of about $+3 \mathrm{~V}$, this DAQ setting implies that a measurement range of slightly above 2000 counts is accessible, which refers to about 11 bits maximum resolution. The electronical noise level of the camera head without the MCP detector was determined by illuminating a mechanical slit with light from a stabilized tungsten ribbon lamp and imaging the slit onto the input side of the Gen I light amplifier. Recording data with a spectra rate of 1000/s, the time evolution of the measured signal from a single pixel shows random noise with a size of \pm 1.5 counts. A more crucial point is the experimental confirmation of the linearity and dynamic range of the complete MCP detector and camera system, since a reproducible VUV/XUV light source with a widely adjustable and known output intensity is not readily available. We performed an indirect test of the linear range by operating the detector with a near-normal incidence VUV spectrometer (McPherson type 234, with concave 2400/mm grating) and monitoring the line intensity ratio between a strong $(58.4 \mathrm{~nm})$ and a weak $(53.4 \mathrm{~nm}) \mathrm{He}$ I line intensity emitted from a stable hollow cathode discharge of Kock-type. ${ }^{17}$ Varying the MCP amplification step by step via changing the MCP voltage, a large range of output signal intensities is easily accessed and possible linearity problems would become noticeable via a nonlinear development of the 


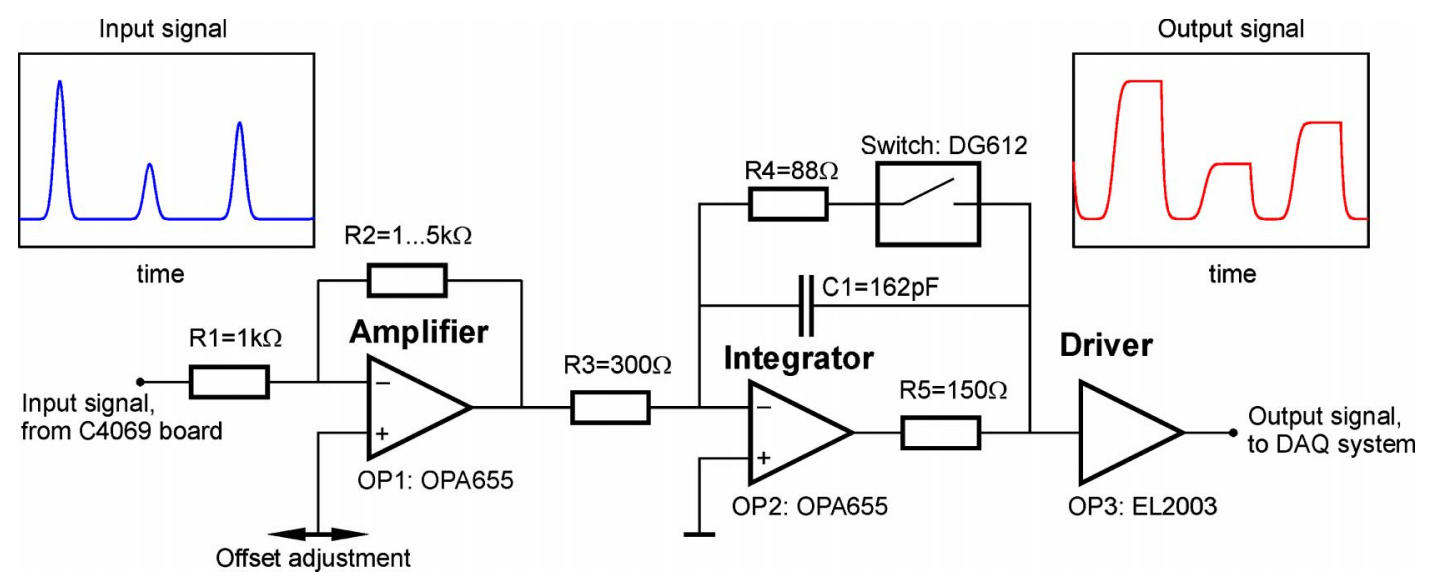

FIG. 2. (Color online) Scheme of the switched integrator board.

measured intensity ratio between both spectral lines. Within the accuracy level as given by the stability range of the hollow cathode discharge (typical $\pm 10 \%$ ), no deviation from linearity is found up to a signal level of 2000 counts, which is in agreement with the estimation of the linearity range given above. From these test results we conclude, that the detector system described here allows to perform linear VUV intensity measurements with a dynamic range for the single pixel of more that 10 bits.

The full spectrum of the VUV intensity from the hollow cathode discharge burning in helium was measured using the detector system with a survey spectrometer ${ }^{3,4}$ of the type survey poor resolution extended domain (SPRED) equipped with a toroidally shaped grating with 450 grooves per $\mathrm{mm}$ (in the following denoted as SPRED-A) and an entrance slit of $50 \mu \mathrm{m}$ width and $4 \mathrm{~mm}$ height. In this experiment the exit aperture of the hollow cathode source with $1.2 \mathrm{~mm}$ diameter was located at a distance of $889 \mathrm{~mm}$ from the entrance slit of the spectrometer, so that only a small fraction of the etendue of the spectrometer was used. A sample spectrum is shown in Fig. 3, where the He I lines at 58.4 and $53.4 \mathrm{~nm}$ as well as the Lyman series of the hydrogen-like He II lines $(30.4 \mathrm{~nm}$ and below) are visible.

The measured FWHM line width amounts to $0.35 \mathrm{~nm}$, which corresponds to 3.5 pixels. This figure can be explained by the optical imaging as well as the additional broadening caused by the detector. The illumination of the entrance slit

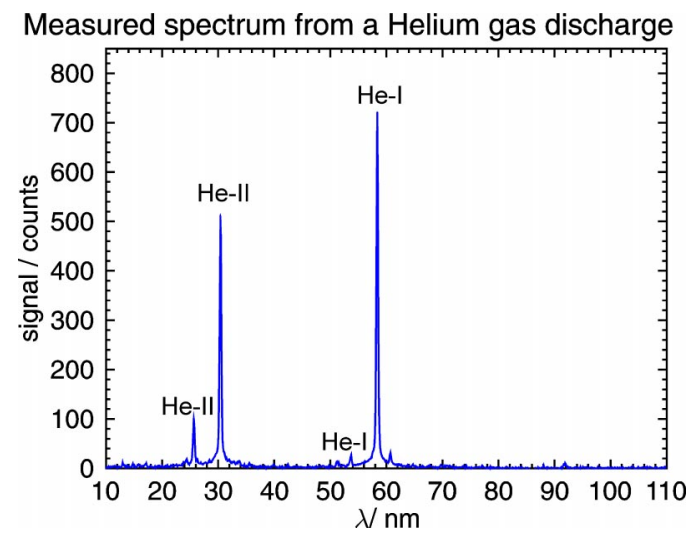

FIG. 3. (Color online) SPRED-A spectrum from a helium hollow cathode discharge. with the light from the point-like light source leads to a slightly curved slit image on the MCP surface with an effective FWHM width of about 70-80 $\mu \mathrm{m}$, which is a factor of 1.5 larger than the width of the entrance slit used. The subsequent demagnification by the $40 / 25$ fiber taper would convert this figure to a slit image width in the order of 2 pixels in the output plane of the fiber taper. The different contributions to the achievable resolution of the detector are estimated as follows (FWHM widths): MCP detector including screen $60-80 \mu \mathrm{m}$, broadening by the fibers and the fiberfiber transitions $20 \mu \mathrm{m}$, spot size of the Gen I light amplifier $40 \mu \mathrm{m}$, linear array resolution $25 \mu \mathrm{m}$. A step by step calculation of the root mean square values along the detector setup yields an estimated figure for the line width of $80-85 \mu \mathrm{m}$, corresponding to a width of about 3.5 camera pixels, which is in agreement with the measured value. We conclude that a point-like input event at the MCP input side would lead to a FWHM width of about 3 pixels measured by the detector system.

A sample spectrum from a TEXTOR discharge with pure ohmic heating measured by the SPRED-A spectrometer with a radial sightline near the horizontal midplane is shown in Fig. 4. Many prominent spectral lines from different plasma impurity species and ionization stages can be clearly distinguished. However, in the spectral region below $30 \mathrm{~nm}$ the large number of spectral lines and the limited spectral reso-

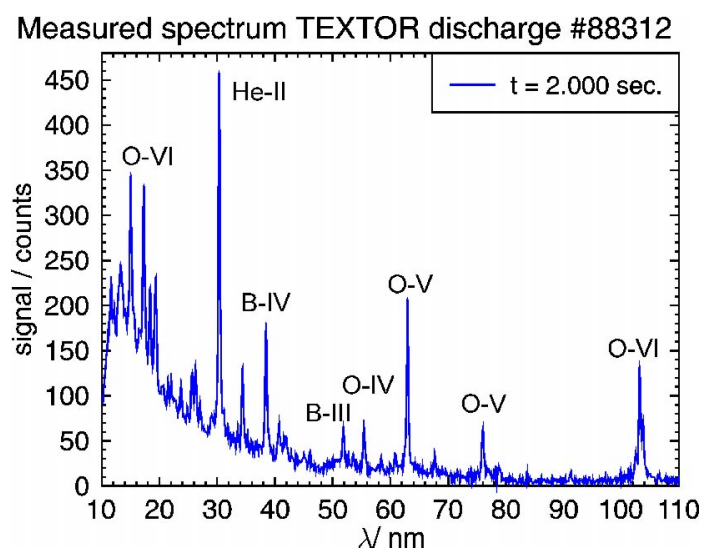

FIG. 4. (Color online) SPRED-A spectrum from TEXTOR discharge No. 88312. 
lution of the SPRED-A instrument impose a severe limitation for line separation and lead to a broad background structure visible in the spectrum. The measured line width in the spectrum from the TEXTOR plasma corresponds to a width of about 4 pixels, which is slightly larger than the value given above for the hollow cathode discharge. This decrease in resolution originates from the fact that the TEXTOR plasma illuminates a much larger solid angle of the spectrometer than the hollow cathode discharge, which leads to an additional broadening of the line width due to aberrations in the optical imaging of the entrance slit onto the MCP by the grating.

In summary, the new detector system allows to operate VUV spectrometers with a continuous spectra readout rate of 1000 per seconds at a full dynamic range of 10 bit, which allows to routinely investigate transient phenomena, e.g., in fusion plasmas.

The authors gratefully acknowledge the skillful assistance of A. Scholl, G. Telemann, D. Brinkmann, and N. Sandri in the preparation of the electronics and mechanics for the camera heads.
${ }^{1}$ R. C. Isler, Nucl. Fusion 24, 1599 (1984).

${ }^{2}$ K. H. Behringer et al., Nucl. Fusion 26, 751 (1986).

${ }^{3}$ R. J. Fonck, A. T. Ramsey, and R. V. Yelle, Appl. Opt. 21, 2115 (1982).

${ }^{4}$ B. C. Stratton, R. J. Fonck, I. K. Jaehnig, and A. T. Ramsey, Rev. Sci. Instrum. 57, 2043 (1986).

${ }^{5}$ J. L. Schwob, A. W. Wouters, S. Suckewer, and M. Finkenthal, Rev. Sci. Instrum. 58, 1601 (1987).

${ }^{6}$ S. A. Cohen, J. L. Cecchi, and E. S. Marmar, Phys. Rev. Lett. 35, 1507 (1975).

${ }^{7}$ K. Krieger, G. Fussmann, and the ASDEX Team, Nucl. Fusion 30, 2392 (1990).

${ }^{8}$ M. A. Graf et al., Rev. Sci. Instrum. 66, 636 (1995).

${ }^{9}$ M. Mattioli, C. De Michelis, and A. L. Pequet, Nucl. Fusion 38, 1629 (1998).

${ }^{10}$ W. Biel et al., Eur. Conference Abstracts 25A, 1389 (2001).

${ }^{11}$ W. Biel et al., Eur. Conference Abstracts 23J, 657 (1999).

${ }^{12}$ W. L. Hodge, B. C. Stratton, and H. W. Moos, Rev. Sci. Instrum. 55, 16 (1984).

${ }^{13}$ W. Biel et al., Eur. Conference Abstracts 26A, P-5.089 (2002).

${ }^{14}$ N. C. Hawkes and N. J. Peacock, Rev. Sci. Instrum. 57, 2035 (1986).

${ }^{15}$ B. E. Chapman et al., Appl. Opt. 33, 4214 (1994).

${ }^{16}$ A. R. Field, J. Fink, D. R. Fussmann, and U. Wenzel, Rev. Sci. Instrum. 66, 5433 (1996)

${ }^{17}$ K. Danzmann, M. Günther, M. Kock, and M. Kühne, Appl. Opt. 27, 4947 (1988). 